\title{
Clinical experience with gamma knife stereotactic radiosurgery in the management of vestibular schwannomas secondary to type 2 neurofibromatosis
}

\author{
J G Rowe, M W R Radatz, L Walton, T Soanes, J Rodgers, A A Kemeny
}

See end of article for authors' affiliations

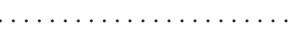

Correspondence to: Mr J Rowe, National Centre for Stereotactic Radiosurgery, Royal Hallamshire Hospital, Glossop Road, Sheffield S10 2JF, UK;

Jeremy.Rowe@sth.nhs.uk

Received

3 October 2002

Accepted in final revised

form 5 April 2003

\begin{abstract}
Objective: To evaluate the results of stereotactic radiosurgery treating vestibular schwannomas secondary to type 2 neurofibromatosis.

Methods: A retrospective review of 122 type 2 neurofibromatosis vestibular schwannomas consecutively treated in 96 patients. Tumour control was assessed by recourse to surgical intervention, by serial radiological imaging, and by the calculation of relative growth ratios in patients $(n=29)$ habouring untreated contralateral tumours to act as internal controls. Hearing function was assessed with Gardner-Robertson grades and with averaged pure tone audiogram thresholds. Other complications are detailed.

Results: Applying current techniques, eight years after radiosurgery it was estimated that $20 \%$ of patients will have undergone surgery for their tumour, $50 \%$ will have radiologically controlled tumours, and in $30 \%$ there will be some variable concern about tumour control, but up to that time they will have been managed conservatively. Relative growth ratios one and two years after treatment indicate that radiosurgery confers a significant $(p=0.01)$ advantage over the natural history of the disease. Analysis of these ratios beyond two years was precluded by the need to intervene and radiosurgically treat the contralateral control tumours in more than $50 \%$ of the cases. This growth control was achieved with $40 \%$ of patients retaining their Gardner-Robertson hearing grades three years after treatment, $140 \%$ having some deterioration in grade, $20 \%$ becoming deaf). Pure tone audiogram results suggest some progressive long term hearing loss, although interpretation of this is difficult. Facial and trigeminal neuropathy occurred in $5 \%$ and $2 \%$.

Conclusions: Radiosurgery is a valuable minimally invasive alternative treatment for these tumours. For most patients, it controls growth or defers the need for surgery, or both. There is a price in terms of hearing function, although this may compare favourably with the deafness associated with the natural history of the disease, and with surgery. In deciding on therapy, patients should be aware of this treatment option.
\end{abstract}

T he dilemmas treating type 2 neurofibromatosis (NF2) vestibular schwannomas (VS, acoustic neuromas) are well recognised. These patients become deaf, and frequently develop other tumours and disabilities. ${ }^{1}$ Clinical management aims to minimise and defer this morbidity. Surgery, the mainstay of active treatment, has significant associated morbidity and mortality, reflected by the move towards increasingly conservative management strategies.

Radiosurgery, in contrast, is minimally invasive, with very different potential complications, most importantly the concern of malignant transformation. What radiosurgery offers the NF2 patient is therefore very different from surgery. This situation has been complicated by our own assessment that, like surgery, ${ }^{2}$ radiosurgery is less effective in treating NF2 VS compared with sporadic tumours (personal communication). Only three radiosurgery groups have published series on this, one supporting this notion ${ }^{3}$ and two reporting results closer to the general experience with VS. ${ }^{45}$ Even if radiosurgery is less effective in NF2 when compared with sporadic tumours, it, like surgery, may have a valuable role in helping these patients. In an attempt to define this role, we report here our total experience treating this condition.

\section{METHODS}

\section{Patient details}

From July 1986 to December 2000, 123 treatments were undertaken for 122 VS in 96 patients with NF2. Four families contributed 10 patients and 17 tumours to the series. Seventy patients had a single VS treated. Twenty six patients had bilateral VS treated, (eight as a single treatment, 18 as staged procedures with a mean time interval of 32 (29) months between treatments). One patient underwent radiosurgery for a right sided tumour, the left side being treated eight months later, with a second radiosurgical treatment to the right side after a further 18 months.

The sex distribution was equal. Mean (SD) age at treatment was 28.9 ( 11.6$)$ years (range $11-59$ years). Mean age at presentation was 24.1 (11.1) years (range 7-53). Twenty seven patients $(28 \%)$ fulfilled the criteria of early presentation, with intracranial disease other than VS, and spinal involvement, for the severe Wishart subtype of NF2.

To permit outcome analysis, in patients undergoing bilateral radiosurgical treatments, each tumour is regarded as an individual entity with its own effects on hearing and cranial nerve function. In patients where VS treatment was combined with treatment of another tumour, these other tumours were discounted from the analysis.

Of the 122 tumours, radiosurgery was performed as the initial treatment in 97 cases, 20 having undergone one previous resection, and five tumours multiple resections. Thirty eight patients had previously undergone surgery to the contralateral

Abbreviations: NF2, type 2 neurofibromatosis; VS, vestibular schwannomas 
Table 1 Morbidity before radiosurgery

\begin{tabular}{llll}
\hline & \multicolumn{3}{c}{ Morbidity before radiosurgery } \\
\cline { 2 - 4 } & Overall & $\begin{array}{l}\text { No previous } \\
\text { surgery }\end{array}$ & $\begin{array}{l}\text { Previous } \\
\text { surgery }\end{array}$ \\
\hline Hearing & 67 & 66 & 1 \\
Gardner-Robertson I-II & 67 & 15 & 2 \\
III-IV & 17 & 16 & 22 \\
V & 38 & 4 & 16 \\
Facial nerve palsy & 20 & 11 & 5 \\
Trigeminal neuropathy & 16 & & \\
\hline $\begin{array}{l}\text { Deafness and the presence of facial nerve palsies were both } \\
\text { significantly (p=0.001) related to previous surgery. Included in the 11 } \\
\text { cases of trigeminal neuropathy with no previous surgical intervention } \\
\text { are four patients who harboured trigeminal neuromas. }\end{array}$
\end{tabular}

side once, and on multiple occasions in six cases. Details of pre-radiosurgical morbidity are summarised in table 1 , deafness and facial nerve palsies being significantly $(\mathrm{p}<0.001)$ associated with previous surgery.

Radiosurgery was undertaken in 82 of the cases $(67 \%)$ because of tumour growth, in 41 cases $(34 \%)$ progressive hearing loss also being a factor. Of the tumours without verified growth, the treatment decision was influenced by hearing loss in 19 cases $(51 \%)$, by tumour size being close to $3 \mathrm{~cm} \mathrm{(a}$ limit for efficacious radiosurgery) in 15 cases $(40 \%)$, and in three cases as a planned adjunct to surgery for a significant residual tumour mass. (These considerations are not mutually exclusive.)

With bilateral tumours, the choice of which tumour to irradiate was determined by disease progression, treating the larger and/or faster growing tumour and the deafer ear. Radiosurgery was only used on a tumour in the only hearing ear, if that hearing was deteriorating or if the tumour was actively growing.

\section{Radiosurgical technique and dose protocols}

The use of the Leksell Gamma Knife (Elekta, Sweden) treating VS is well described. ${ }^{67}$ During the time encompassed by this study, there were significant advances in imaging and treatment planning, and in dose protocols. ${ }^{8}$ To allow for this, the patients are divided into three groups (table 2 ): an early group (group 1, 1986-87) treated with 25 Gy, an intermediate group (group 2, 1988-94) prescribing 17.5-20 Gy, and a late group (group 3, from 1993) closest to our current practice, with MRI localisation, multiple isocentres, and conformal planning, prescribing mainly 13-15 Gy, with some further reductions down to $10 \mathrm{~Gy}$ in pursuit of hearing preservation. Prescriptions were to the $50 \%$ isodose. While we have included all of these cases, this paper concentrates on the group 3 patient results, as this most accurately reflects what current radiosurgery has to offer.

\section{Patient assessment and follow up}

Clinical, radiological, and audiometric examinations were performed at six months and then yearly after radiosurgery.
Reflecting the referral area from which these patients came, these examinations were mainly performed locally in their referring hospitals. When information was incomplete, this was actively sought from physicians involved with the patient's care.

The efficacy of radiosurgery in controlling tumour growth is not easy to ascertain. Included therefore are three measures of tumour control: the rate at which surgical resection was undertaken, the results of serial imaging, and where applicable relative growth ratios. The results of serial imaging were assessed qualitatively, reflecting the problems in making absolute measurements when tumours are scanned with different modalities and protocols.

The exception to this approach is the use of relative growth ratios. These ratios consider tumour growth relative to an untreated contralateral VS acting as an internal control. ${ }^{9}$ This measurement only applies to those patients with an untreated contralateral tumour. If $\mathrm{V}_{0}$ is the volume of the tumour being irradiated at the time of treatment, and $\mathrm{C}_{0}$ the volume of the contralateral tumour, and $V_{t}$ and $C_{t}$ the respective volumes after a given period of time $(\mathrm{t})$, then

Relative growth ratio $=\mathrm{V}_{0} / \mathrm{C}_{0} \times \mathrm{C}_{\mathrm{t}} / \mathrm{V}_{\mathrm{t}}$

expressed as a percentage. A figure of $100 \%$ suggests that radiosurgery has no demonstrable effect, both tumours growing at the same rate. A figure above $100 \%$ suggests that the treated tumour is shrinking, or not growing as fast as the other side. A value less than $100 \%$ suggests that radiosurgery has failed to control growth. As each of two scans contribute to both the numerator and the denominator, use of the ratio avoids concerns about errors from different scanning techniques.

Hearing was classified using Gardner-Robertson grades. ${ }^{10}$ Additionally patients with serial pure tone audiogram records, had threshold values between $1 \mathrm{kHz}$ and $4 \mathrm{kHz}$ averaged, (a similar approach to surgical series ${ }^{2}$ ). These average thresholds were plotted against time relative to radiosurgery. Individual patients showed very different temporal patterns of hearing loss, four examples being plotted to illustrate this. To generate an overall picture, the gradient of hearing loss for individual patients was calculated, averaged for the whole patient group (giving a mean and standard deviation for the hearing loss occurring at different times relative to radiosurgery), and summed to give a mean cumulative hearing loss.

Facial weakness was graded using the House-Brackmann scale. ${ }^{11}$ Any subjective sensory change was included as trigeminal neuropathy whether or not objective signs were present. Evidence of worsening vestibular symptoms or brain stem function was also sought.

Regarding follow up, patients left this study for one of three reasons: the tumour was resected, the patient died, or the patient was lost to follow up. The rates of tumour resection are specifically considered, representing a distinct end point. The mortality rate is detailed separately. With time and worsening disability, it is understandable that patients withdraw from

Table 2 Clinical details for the different patient groups defined by dose prescribed to the $50 \%$ isodose

\begin{tabular}{llllllll}
\hline Group & $\begin{array}{l}\text { Number } \\
\text { of tumours }\end{array}$ & $\begin{array}{l}\text { Time } \\
\text { period }\end{array}$ & $\begin{array}{l}\text { Dose } \\
\text { range (Gy) }\end{array}$ & $\begin{array}{l}\text { Mean (SD) } \\
\text { dose (Gy) }\end{array}$ & $\begin{array}{l}\text { Mean } \\
\text { number } \\
\text { of isocentres }\end{array}$ & $\begin{array}{l}\text { Tumour } \\
\text { volume } \mathrm{cm}^{3}\end{array}$ & $\begin{array}{l}\text { Follow up in } \\
\text { months mean } \\
\text { (SD: range) }\end{array}$ \\
\hline 1 & 13 & $1986-87$ & 25 & $25(0)$ & $1.5(0.7)$ & $12.2(16.7)$ & $82(59: 4-136)$ \\
2 & 17 & $1988-94$ & $17.5-20$ & $17.8(0.8)$ & $1.7(1.0)$ & $3.8(4.0)$ & $89(48: 9-154)$ \\
3 & 92 & From 1993 & $10-16$ & $13.4(1.6)$ & $5.4(2.5)$ & $4.6(4.3)$ & $38(23: 6-89)$ \\
Overall & 122 & $1986-2000$ & $10-25$ & $15.2(4.0)$ & $4.5(2.7)$ & & $50(38: 4-154)$ \\
\hline
\end{tabular}

With experience, dose reduction was undertaken to decrease cranial nerve complications. At the same time patient selection evolved with smaller tumours being treated, and planning became more sophisticated, more isocentres being used. Overall, the median follow up for all patients was 51 months. 


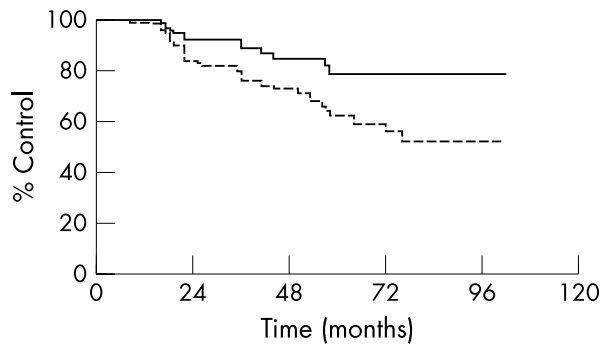

Figure 1 Kaplan-Meier plots illustrating the rate at which surgery is undertaken (solid line), and control defined by surgical intervention, radiological growth, or clinical concern (broken line). Results are for the current low dose treatment group.

clinic appointments and are lost to follow up. This loss will affect the quality of the data. To allow for this, follow up times are calculated for surviving tumours, excluding patient deaths and tumour resections from these data.

Values are expressed as mean (SD). Statistical comparisons use paired and unpaired $t$ tests, and $\chi^{2}$ tests as appropriate. Rates of surgical resection and radiological tumour control rates are represented with Kaplan-Meier plots, log rank tests being used to compare these survival curves.

\section{RESULTS}

During this study, we know that 15 of the 96 patients died on average 50 (42) months after radiosurgery. All deaths were the consequence of NF2, three patients dying of neurosurgical complications. Six further patients were severely disabled with end stage neurofibromatosis, and withdrew from follow up. As other patients were lost to follow up without clearly defined reasons, these figures underestimate the morbidity and mortality experienced by this patient group.

Figure 1 illustrates the rate at which surgical resection was undertaken after radiosurgery for the current practice group 3 patients. As $75 \%$ of the total patient series were in group 3, this plot closely resembles the results for the three groups combined. There were no statistically significant differences between the curves for the three groups. The group 3 plot suggests that $79 \%$ of patients avoid surgery for eight years after radiosurgery.

The Kaplan-Meier plot is recalculated combining patients undergoing surgery with those whose imaging showed tumour growth, and those with increasing symptoms - that is, to include patients with a loss of tumour control radiologically or clinically. This estimates that $52 \%$ of tumours are controlled at eight years. These survival curves were not significantly different between the different dose groups.

Thirty four patients had previously untreated contralateral tumours, there being adequate data to generate relative growth ratios in 29 of these (table 3, fig 2). Of the 29 patients, 15 had the contralateral schwannoma treated radiosurgically on average 27 (14) months after the first side. In two patients

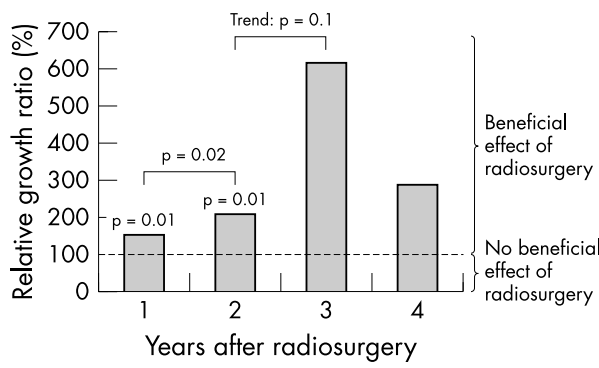

Figure 2 Changes in relative growth ratios are shown based on data from 29 patients with untreated contralateral control tumours. Values greater than $100 \%$ indicate a beneficial effect of radiosurgery. This was seen one and two years after treatment $(p=0.01)$. Thereafter growth of the contralateral control tumour necessitated radiosurgical intervention in more than $50 \%$ of the patients. Treating the fastest growing control tumours, and removing these patients from the analysis, reduces numbers and statistical significance, and would be predicted to decrease the ratio, this being seen in the fourth year.

radiosurgery failed to control growth, surgery being performed after 34 (1) months. Three patients died on average three years after treatment. Reflecting this, the data mostly relate to the first two years after radiosurgery, when the ratio is significantly increased $(p=0.01)$, radiosurgery having a beneficial effect. Examining paired data, there was a significant increase in the ratio between the first and second year $(p=0.02)$, this trend continuing between the second and third years $(p=0.1)$. By three years the need to treat the contralateral tumour reduces patient numbers, statistical significance being lost.

The dose reduction from group 1 to group 3 increased hearing preservation $(\mathrm{p}=0.05)$. With current doses, about $40 \%$ have preserved hearing (unchanged Gardner-Robertson grade) three years after radiosurgery, $40 \%$ having some reduction in grade, $20 \%$ becoming completely deaf. Of the 25 tumours that had undergone surgery, only three, all treated with deliberately cautious subtotal resections, had hearing (one grade II, two grade III). Of these, one group 1 and one group 3 patient became deaf, one group 3 patient preserving hearing (table 4).

Thresholds were averaged from 190 pure tone audiograms examining 46 hearing ears in 35 patients, spanning a period six years before to 12 years after radiosurgery. Forty of these 46 tumours (87\%) relate to our current group 3 practice. Between patients there was marked heterogeneity (fig 3), one patient exceptionally showing improvement, others with long term preservation, and others more rapid deterioration. Figure 4 attempts to provide an overview, averaging and summing the gradient of hearing loss to give a mean cumulative value. Most of the audiograms are performed around the time of radiosurgery, few patients contributing to the extreme time points, this being represented by the bar chart and interquartile range.

The dose reduction also reduced the incidence of facial nerve palsies and trigeminal neuropathy ( $p=0.001$, table 5$)$. In

Table 3 Mean (SD) relative growth ratios from 29 patients with untreated contralateral tumours acting as internal controls

\begin{tabular}{|c|c|c|c|c|c|}
\hline & 1 Year & & 2 Years & 3 Years & 4 Years \\
\hline Relative growth ratio & $151(72) \%$ & & $205(120) \%$ & $616(757) \%$ & $285(245) \%$ \\
\hline Number & 19 & & 16 & 7 & \\
\hline Significance from $100 \%$ & $p=0.01$ & & $p=0.01$ & NS & NS \\
\hline Significant rise & & $p=0.02$ & & & \\
\hline
\end{tabular}

Values in excess of $100 \%$ indicate a beneficial effect of radiosurgery. This is seen at one and two years, the increase between the first and second year also being significant $(p=0.02)$, this trend continuing into the third year. Fifteen of the 29 patients subsequently required radiosurgery to control the tumours, because they were growing. This occurred on average 27 (14) months after the initial treatment. Treating these tumours and removing these patients from the analysis, decreases the numbers, and with this statistical significance is lost. 
Table 4 Effect of radiosurgery on hearing assessed by Gardner-Robertson grades

\begin{tabular}{|c|c|c|c|c|c|c|c|}
\hline \multirow[b]{2}{*}{$\begin{array}{l}\text { Dosimetry } \\
\text { group }\end{array}$} & \multirow[b]{2}{*}{$\begin{array}{l}\text { Incomplete } \\
\text { data }\end{array}$} & \multicolumn{3}{|c|}{ Before treatment } & \multicolumn{3}{|l|}{ After treatment } \\
\hline & & $\begin{array}{l}\text { Grade } \\
\text { I-II }\end{array}$ & $\begin{array}{l}\text { Grade } \\
\text { IIIIIV }\end{array}$ & $\begin{array}{l}\text { Grade } \\
\mathrm{v}\end{array}$ & $\begin{array}{l}\text { Preserved } \\
\text { hearing }\end{array}$ & $\begin{array}{l}\text { Deterioration } \\
\text { in hearing }\end{array}$ & Deaf \\
\hline $\begin{array}{l}\text { Group } 1 \\
(n=13)\end{array}$ & 1 & 6 & 3 & 3 & $\begin{array}{l}2(22 \%) \\
(52(6) \text { months) }\end{array}$ & $\begin{array}{l}1 \text { (11\%) } \\
\text { (7 months) }\end{array}$ & $9(67 \%)$ \\
\hline $\begin{array}{l}\text { Group 2 } \\
(n=17)\end{array}$ & 2 & 9 & 1 & 5 & $\begin{array}{l}2(20 \%) \\
\text { (74 (101) months) }\end{array}$ & $\begin{array}{l}6(60 \%) \\
\text { (43 (15) months) }\end{array}$ & $7(20 \%)$ \\
\hline $\begin{array}{l}\text { Group } 3 \\
(n=92)\end{array}$ & 5 & 49 & 12 & 26 & $\begin{array}{l}23(38 \%) \\
\text { (40 (25) months) }\end{array}$ & $\begin{array}{l}26(42 \%) \\
(41 \text { (21) months) }\end{array}$ & $38(20 \%)$ \\
\hline
\end{tabular}

Preserved hearing refers to an unchanged grade before and after treatment. Deterioration is defined as any decline in hearing grade other than becoming completely deaf (grade V). Percentages refer to the proportion of patients with discernible (grade I-IV) hearing before treatment who have preservation of, deterioration in, or no hearing after treatment, with follow up details of when the assessment was made. Dose reduction from group 1 to group 3 was associated with a significant improvement in hearing preservation $(p=0.05)$.

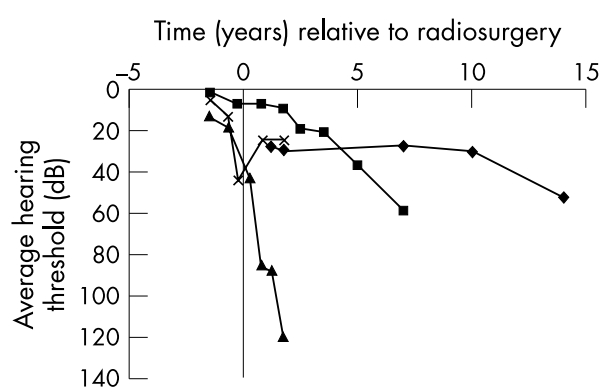

Figure 3 Variability between individual patients in hearing thresholds and their response to radiosurgery is illustrated. Threshold values are pure tone audiogram results averaged over $1-4 \mathrm{kHz}$ and plotted in relation to the time of treatment. One of these patients shows some late deterioration, having had good hearing preservation for 10 years. Only one patient in the entire series experienced improvement in hearing. Deterioration when it occurs does so at very variable rates.

Table 5 Facial and trigeminal nerve complications after radiosurgery

\begin{tabular}{lllll}
\hline & $\begin{array}{l}\text { Incomplete } \\
\text { data }\end{array}$ & $\begin{array}{l}\text { Intact or } \\
\text { unchanged }\end{array}$ & Palsy & Improved \\
\hline Facial nerve complications & & & \\
Group 1 $(n=13)$ & - & 5 & 8 & \\
Group 2 $(n=17)$ & 1 & 14 & 2 & \\
Group 3 $(n=93)$ & 3 & 83 & 7 & \\
Trigeminal nerve complications & & & \\
Group 1 $(n=13)$ & - & 7 & 6 & \\
Group 2 $(n=17)$ & 1 & 15 & 1 & 1 \\
Group 3 $(n=93)$ & 7 & 83 & 2 & 1 \\
\hline
\end{tabular}

Classified as incomplete trigeminal nerve data are four patients who could not be assessed because of the presence of trigeminal neuromas. Dose reduction significantly $(p=0.001)$ reduced both facial and trigeminal nerve complications.

the group 3 patients, facial nerve function worsened in seven cases $(8 \%)$. Three of these were transient, two were exacerbations of pre-existing palsies (a grade $2 / 3$ and 4 palsy becoming grade 4 and 5 weaknesses), two patients developing new grade 2-3 palsies. The incidence of persisting facial palsy with current doses is less than 5\%. Excluding trigeminal neuromas, two group 3 patients ( $2 \%$ ) developed new sensory symptoms, one patient's pre-existing neuralgia improving.

Non-specific vestibular symptoms, (balance problems, tinnitus, earache or a "fullness in the ear", etc), were only reported by seven patients (6\%). No patients developed brain stem symptoms related to radiosurgery.

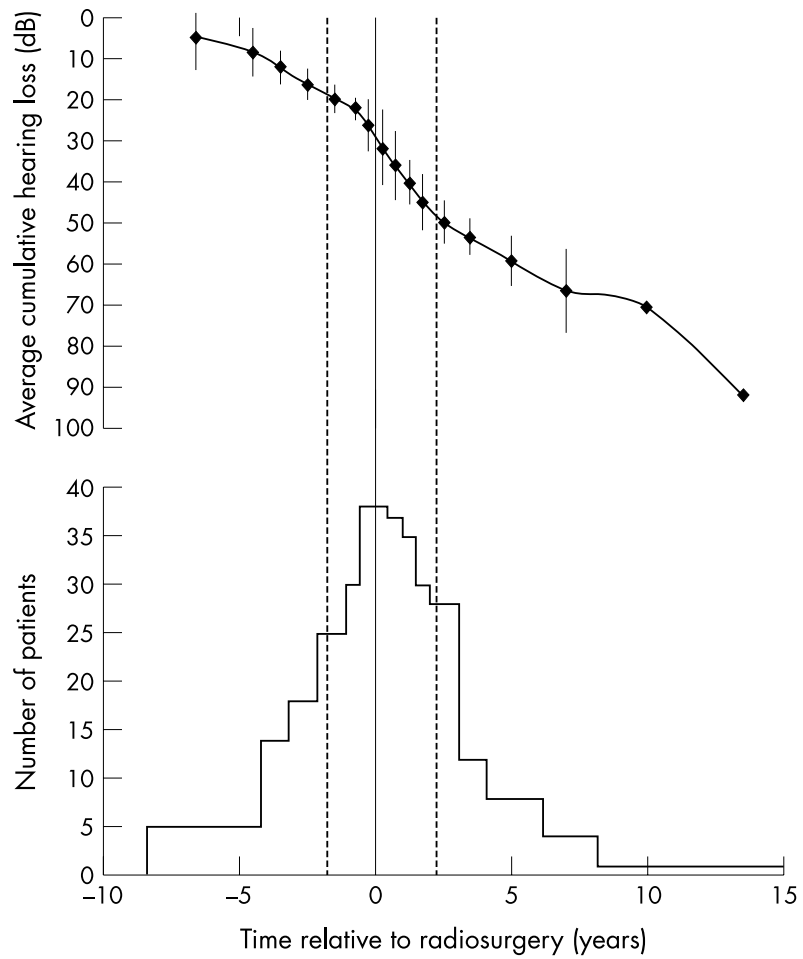

Figure 4 Pure tone audiogram results represented as an averaged cumulative hearing loss. For reasons discussed in the text, this representation may be a falsely pessimistic view of the changes in hearing function, particularly at the extremes of the curve where comparatively little patient data may skew the results. To address this, the histogram illustrates the amount of patient data contributing to the curve, the vertical broken lines being the interquartile range of this. Error bars represent the standard deviation of the hearing loss.

\section{DISCUSSION}

\section{Tumour control}

Tumour control is difficult to define. The simplest measure of control, or loss of it, is the rate at which surgical resection is undertaken. This is important because patients undergo radiosurgery with the specific aim of avoiding surgery. In a sense it can be answered precisely: $79 \%$ of patients avoid surgery for eight years.

This however is falsely simplistic. While surgery is a clear event, lending itself to a Kaplan-Meier plot, the decision to undergo it is anything but. There may be significant reticence both from the patient and the surgeon. An extreme example of these issues is afforded by one of our patients dying of pneumonia 14 months after radiosurgery. Factors in his death 
included end stage NF2, hypostasis from paraparesis and spinal disease, and concern about growing infratenorial and supratentorial tumours. With decreasing therapeutic options, he withdrew from radiological follow up and neurosurgical review, and there is no record of his bulbar function. In terms of freedom from surgical resection, this represents a radiosurgical success; in terms of radiological tumour control, the tumour was not imaged; in terms of growth control beyond the natural history of the disease, it is impossible to ascertain, as it is not clear which factors contributed to his death. We have raised the issue of assessing tumour control in unilateral VS, ${ }^{8}$ although the concerns are much more extreme and potentially confounding when applied to NF2. To investigate this, we recalculated the Kaplan-Meier plots, including as uncensored observations not only patients undergoing surgery, but those in whom there was any concern about tumour control. This offers a best and worst case scenario. We summarise this, using current doses, within eight years of radiosurgery, $20 \%$ of NF2 patients will undergo surgery, $50 \%$ will have controlled tumours, in 30\% there will have been variable concerns about control, but they will have been managed conservatively.

The time limit is important, not only in reflecting the length of our experience with the current dose protocol, but because one group 2 patient had tumour growth 12 years after radiosurgery. NF2 may differ in this respect from sporadic tumours. Indeed, it is not clear whether this late growth is recurrence of the previously treated tumour, or growth of a new lesion. The clinical implication is that long term follow up is indicated.

Interpreting growth control depends on the natural history of the disease. Establishing this for NF2 is difficult, because of its rarity, its phenotypic heterogeneity, and because of the pressure to intervene when patients deteriorate. The limited estimates available suggest that $75 \%-79 \%$ of NF2 VS are growing. ${ }^{12}{ }^{13}$ The relative growth ratio is an alternative approach to study the natural history. It requires a contralateral untreated tumour, which limits patient numbers, but does provide as perfectly matched a control as is possible. It is not without criticism in that the understanding of tumour suppressor genes in NF2 is that while one genetic mutation is the predisposing factor, a second mutation in the corresponding allele is required for tumourogenesis. The genotype of two tumours in a single NF2 patient may not therefore be identical. In response to this, the more aggressive tumour would be treated first, comparison with the less aggressive tumour underestimating any benefit of radiosurgery. Despite this, figure 2 illustrates the beneficial effects of radiosurgery (table 3). Values of the ratio above $100 \%$ are evidence of a beneficial effect of radiosurgery. One year after treatment, the ratio was significantly $(p=0.01)$ increased. This increased further in the second year, not only the mean ratio being increased $(p=0.01)$, but the increase from year one to year two being significant $(p=0.02)$, most probably reflecting continued growth of the untreated tumour. This trend continued into the third year, although by this stage patients were increasingly having the opposite schwannoma treated, removing them from the comparison. More than $50 \%$ of the patients in this analysis underwent radiosurgery to the contralateral control tumour on average 27 months after the first side was treated. Treating the fastest growing contralateral control tumours (and removing them from the analysis) should decrease the ratio, this being seen by the fourth year. Importantly, included in this relative growth ratio analysis are all patients $(n=29)$ with untreated control tumours and adequate imaging data to permit the calculations, there being no other superselection. Indeed, included is one patient with a malignant tumour (discussed below) that continued to grow despite radiosurgery. While relative growth ratios are more abstract than other measures of tumour control, they take into account the natural history of the disease, and what radiosurgery might achieve in slowing tumour growth even if growth is not controlled.

\section{Hearing}

Our hearing preservation rate of $40 \%$ is poor compared with unilateral tumours, ${ }^{8}$ although interpretation has to consider that $34 \%$ of the NF2 patients were treated because of deteriorating hearing. The problem in advising patients is the individual variability in the response of hearing to radiosurgery. Recovery was only seen in one patient, so patients are never advised to have treatment in the hope that hearing will improve. Long term hearing preservation is achieved in some patients, although as reflected by the Gardner-Robertson grades, most have some continued deterioration.

Figure 4 attempts to average hearing threshold data for the whole group. It demonstrates the progressive hearing loss all too familiar in NF2. The gradient of this appears steepest around the time of radiosurgery, this change developing before treatment, reflecting that treatment is often carried out because of hearing loss. Importantly, most audiograms are carried out around the time of an intervention, or when a patient complains of hearing loss. This probably means that the data around the time of radiosurgery are reliable, but that the extreme time points are not. A concern is that if hearing is either maintained or deteriorates, while audiograms are preferentially performed when there is deterioration, this information will bias the cumulative average hearing loss, overestimating the deterioration in hearing. These issues are illustrated by the patients with the longest audiogram follow up after radiosurgery. His data are included in figure 3, where there is hearing preservation lasting more than 10 years. His late deterioration of $20 \mathrm{~dB}$ after 10 years is added to a cumulative hearing loss for the whole group. This is obviously questionable. In an attempt to resolve these concerns, we have included a bar chart to reflect the amount of patient data with lines indicating the interquartile range.

\section{Other complications}

The persisting facial nerve palsy rate of $5 \%$ reported here is greater than the $1 \%$ in our unilateral schwannoma series. ${ }^{8}$ This perhaps is to be expected, given the anatomical differences in $\mathrm{NF} 2 .{ }^{14}$ Excluding trigeminal neuromas, the risk of trigeminal neuropathy $(2 \%)$ did not appear to be different from our unilateral tumour series. No patients developed brain stem complications attributed to the radiosurgery.

\section{Other treatment series}

Three other groups have published radiosurgery on NF2. ${ }^{3-5} 9$ This series is by far the largest, with the longest follow up, and the worst numerical results. Factors influencing this include how results are calculated, how outcomes are defined, and patient selection. Previous series have used simple proportions, with limited follow up, rather than survival curves. Even patients undergoing surgery are not always deemed to have failed. ${ }^{49}$ There may be some patient selection bias, the two groups with the best results treating older patients with less aggressive forms of NF2. We explore the radiosurgical issues of patient selection in terms of phenotype and tumour size, and dose in a companion paper. ${ }^{15}$

Comparison of radiosurgery with surgery is difficult because they differ in what they entail and aim to achieve. Surgery has the great advantage of creating space. The disadvantage is that it remains a major undertaking with associated morbidity and mortality. The largest series, that of Samii, ${ }^{2}$ describes 120 tumours in 82 patients, two of whom died. A hearing preservation rate (defined as a threshold above $90 \mathrm{~dB}$ ) of $36 \%$ is quoted; $57 \%$ having normal facial nerve function; $2 \%-3 \%$ new trigeminal symptoms; and 5\% caudal cranial nerve palsies. Baldwin et al were more pessimistic, regarding the prognosis for hearing after surgery as being "dismal" ${ }^{13} \mathrm{~A}$ similarly bleak outlook was reported by physicians attempting a comprehensive review of the British NF2 patients, who concluded that hearing was preserved in less than $10 \%$, with some 
degree of facial nerve dysfunction in more than $70 \%$ of patients postoperatively. ${ }^{1}$

\section{Hearing preservation strategies}

In considering the relative roles of surgery and radiosurgery for hearing presevation, there are two other issues: the use of combined approaches and auditory brain stem implants. By combined approaches, we refer to planned subtotal resections of large schwannomas followed by radiosurgery for the residual. In our experience, hearing was only preserved in one of two cases treated with our current dose protocol. The use of auditory brain stem implants is not part of our current practice. Even in the largest NF2 series, only $7 \%$ of patients receive these devices, ${ }^{16}$ although their use may become more widespread. While it has been suggested that radiosurgery could cause scarring, making subsequent implants more difficult or less effective, the current literature would not support this. ${ }^{17}$

\section{Risk of malignancy}

The other criticism levelled at radiosurgery, is the concern about malignant transformation. In our department's total experience treating $700 \mathrm{VS}$, we have encountered one patient, who did have NF2, with malignant features confirmed histologically. This patient has been reported in detail elsewhere. ${ }^{18}$ This tumour was rapidly growing, increasing in volume 15 -fold in the two years before radiosurgery. Over the two years after radiosurgery the tumour increased a further threefold. This necessitated resection, histology showing mitotic activity and cellular pleomorphism. The tumour recurred, the patient subsequently dying. Importantly, the tumour behaved atypically before radiosurgery, there being no evidence that the radiosurgery caused this, or was responsible for the malignant cellular features seen histologically.

While we understand concerns associating radiation with tumourogenesis, different forms of radiation may have different biological effects. The risk of causing genetic mutations might be increased with lower radiation doses, damaging DNA without killing cells. One might therefore predict more effects with conventional fractionated radiotherapy, treating large volumes of brain with repeated small doses. Stereotactic radiosurgery is the antithesis of this, minimising tissue exposure, and using a single high dose treatment. Reviewing the world literature, reports of malignant VS remain very rare despite the interest in these cases. We accept, to answer this question definitively, would require lifelong follow up of these patients, and we are currently investigating the feasibility of a mortality database to acheive this. On the data available to us, the risk of malignant transformation seems very low, ${ }^{18}$ less than the risk of death associated with VS surgery in the general, let alone the NF2, population. ${ }^{19}$

\section{Conclusions}

Gamma knife radiosurgery is less efficacious in treating VS secondary to NF2, when compared with sporadic tumours. Despite this, we have no doubt that radiosurgery does offer the NF2 patient an advantage in slowing and/or controlling tumour growth. The relative growth ratio calculations are convincing evidence of this. In practical terms, eight years after radiosurgery, about $20 \%$ of NF2 patients will have required surgery, $50 \%$ will be radiologically controlled, and in $30 \%$ there will be some variable concerns about tumour control, but up to that stage they will have been managed conservatively.

This is achieved with about $40 \%$ of patients preserving their Gardner-Robertson hearing grades, $40 \%$ having some deterioration, and $20 \%$ becoming deaf. The risk of other morbidity is low, with a persisting facial palsy rate of $5 \%$, and trigeminal symptoms in $2 \%$.
The decision of when and how to intervene therapeutically in NF2 remains difficult, because there are no ideal solutions. The options are observation, surgery, and radiosurgery. Observation entails the natural history of the condition, which is one of tumours presenting earlier and growing more aggressively than their sporadic counterparts, associated with progressive deafness and other neurological disabilities. Surgery constitutes a major intervention, with significant morbidity and mortality, concern about which is best reflected by the move towards conservative management. Radiosurgery may offer a valuable, minimally invasive alternative, allowing perhaps half the patients to avoid surgery and a further proportion to defer it. In deciding on management, patients should be aware of what radiosurgery has to offer.

\section{ACKNOWLEDGEMENTS}

The authors wish to express their gratitude to Mr David Forster for his work in establishing radiosurgery in Sheffield, and for his care of many of the patients reported here. The authors also wish to thank Dr Susan Huson for her encouragement and review of the manuscript.

\section{Authors' affiliations}

J G Rowe, M W R Radatz, L Walton, T Soanes, J Rodgers, A A Kemeny, National Centre for Stereotactic Radiosurgery, Royal Hallamshire Hospital, Sheffield, UK

Competing interests: none declared.

\section{REFERENCES}

1 Evans DGR, Huson SM, Donnai D, et al. A clinical study of type 2 neurofibromatosis. QJ Med 1992;304:603-18.

2 Samii M, Matthies C, Tatagiba M. Management of vestibular schwannomas (acoustic neuromas): auditory and facial nerve function after resection of 120 vestibular schwannomas in patients with neurofibromatosis 2. Neurosurgery 1997;40:696-706.

3 Roche PH, Regis J, Pellet W, et al. Neurofibromatosis type 2. Preliminary results of gamma knife radiosurgery of vestibular schwannomas. Neurochirugie 2000:46:339-53.

4 Subach BR, Kondziolka D, Lunsford LD, et al. Stereotactic radiosurgery in the management of acoustic neuromas associated with neurofibromatosis type 2. J Neurosurg 1999;90:815-22.

5 Kida Y, Kobayashi T, Tanaka T, et al. Radiosurgery for bilateral neurinomas associated with neurofibromatosis type 2. Surg Neurol 2000;53:383-9

6 Ganz JC. Gamma knife surgery, a guide for referring physicians. New York: Springer-Verlag, 1993.

7 Kondziolka D, Lunsford LD, McLaughlin MR, et al. Long term outcomes after radiosurgery for acoustic neuromas. N Engl J Med 1998;339: 1426-33

8 Rowe JG, Radatz M, Walton L, et al. Current results with gamma knife stereotactic radiosurgery treating unilateral acoustic neuromas. J Neurol Neurosurg Psychiatry (in press).

9 Linskey ME, Lunsford LD, Flickinger JC. Tumor control after stereotactic radiosurgery in neurofibromatosis patients with bilateral acoustic tumours. Neurosurgery 1992;31:829-39.

10 Gardner G, Robertson JH. Hearing preservation in unilateral acoustic neuroma surgery. Ann Otol Rhinol Laryngol 1988;97:55-66.

11 House WF, Brackmann DE. Facial nerve grading system. Otolaryngol Head Neck Surg 1985;93:184-93.

12 Kishore A, O'Reilly BF. A clinical study of vestibular schwannomas in type 2 neurofibromatosis. Clin Otolarygol 2000;25:561-5.

13 Baldwin D, King T, Chevretton E, et al. Bilateral cerebellopontine angle tumors in neurofibromatosis type 2. J Neurosurg 1991;74:910-15.

14 Jaaskelainen J, Paetau A, Pyykko I, et al. Interface between the facial nerve and large acoustic neurinomas. J Neurosurg 1994;80:541-7.

15 Rowe JG, Radatz M, Walton L, et al. Stereotactic radiosurgery for type 2 neurofibromatosis acoustic neuromas: patient selection and tumour size. Stereotact Funct Neurosurg 2002;79:107-16.

16 Matthies C, Thomas S, Moshrefi M, et al. Auditory brainstem implants: current neurosurgical experiences and perspective. J Laryngol Otol Suppl 2000;27:32-6.

17 Kalamarides M, Grayeli A, Bouccara D, et al. Hearing restoration with auditory brainstem implants after radiosurgery for neurofibromatosis type 2. J Neurosurg 2001;95:1028-33.

18 Bari M, Forster D, Kemeny A, et al. Malignancy in a vestibular schwannoma. Report of a case with central neurofibromatosis treated by stereotactic radiosurgery and surgical excision with a review of the literature. Br J Neurosurg 2002;16:284-9.

19 Samii M, Matthies C. Management of 1000 vestibular schwannomas (acoustic neuromas): surgical management and results with an emphasis on complications and how to avoid them. Neurosurgery 1997;40: 1 1-23. 